\title{
A fresh look into the pathophysiology of ischemia-induced complications in patients with chronic kidney disease undergoing hemodialysis
}

This article was published in the following Dove Press journal: International Journal of Nephrology and Renovascular Disease 13 March 2015

Number of times this article has been viewed

\author{
Patrick M Honore' \\ Rita Jacobs' \\ Elisabeth De Waele' \\ Viola Van Gorp' \\ Jouke De Regt' \\ Olivier Joannes-Boyau ${ }^{2}$ \\ Willem Boer ${ }^{3}$ \\ Herbert D Spapen' \\ 'Intensive Care Department, \\ Universitair Ziekenhuis Brussel,Vrije \\ Universiteit Brussel, Brussels, Belgium \\ ${ }^{2}$ Intensive Care Unit, Haut Leveque \\ University Hospital of Bordeaux, \\ University of Bordeaux 2, Pessac, \\ France; ${ }^{3}$ Intensive Care Department, \\ Ziekenhuis Oost Limburg, Genk, \\ Belgium
}

\begin{abstract}
Recent case reports of acute esophageal necrosis in patients with chronic kidney disease (CKD) undergoing hemodialysis encouraged us to look beyond hypoperfusion/ischemia as a sole explanation for this dramatic complication. At least three intriguing pathways, ie, accumulation of protein-bound toxins, endotoxin translocation, and altered mucosal defense mechanisms, have been proposed to explain the inherent susceptibility of CKD patients to developing ischemia-related and cardiovascular events. Interestingly, all the proposed pathways can be potentially antagonized or attenuated. At present, however, it is not known whether one pathway predominates or if any interaction exists between these pathways. More solid experimental and clinical data are warranted to acquire a better insight into the complex pathogenesis of CKD-associated ischemia.
\end{abstract}

Keywords: chronic kidney disease, ischemia, pathophysiology, cardiovascular events

\section{Introduction}

Acute esophageal necrosis (AEN, also known as "black esophagus") in the absence of ingestion of a caustic or corrosive agent is rarely described in the literature. ${ }^{1}$ Its incidence is estimated to be as low as $0.01 \%$. Although AEN may present as a complication of various pathologic conditions (eg, gastric outlet obstruction, allergic reaction to antibiotics, gastric volvulus, viral infection), ischemia is the main culprit in most cases. ${ }^{1-3} \mathrm{AEN}$ is thought to result from a combination of esophageal ischemia, impaired mucosal barrier function, and chemical injury due to backflow of gastric secretions and contents. ${ }^{1,2,4}$

In a recent report of AEN in a patient with dialysis-dependent chronic kidney disease (CKD), ${ }^{5}$ the authors postulated that AEN was precipitated by repeated episodes of hypovolemic hypoperfusion during hemodialysis. CKD patients undergoing dialysis are indeed particularly prone to ischemia-related complications. First, a majority has underlying diseases (eg, diabetes) characterized by a rapidly progressive atherosclerotic process. Second, CKD itself is associated with enhanced formation of atherosclerotic plaques. Finally, hypovolemic insults of any kind may occur during hemodialysis and result in "acute-on-chronic" hypoperfusion. In addition to these well acknowledged risk factors, new insights regarding ischemia in CKD have recently been elaborated. Here we review some of the most relevant theories.

\section{Accumulation of protein-bound toxins P-cresyl sulfate}

$\mathrm{CKD}$ is characterized by accumulation of so-called "uremic retention solutes". ${ }^{6}$ Colonic microbial metabolism contributes substantially to the production of these solutes.
Intensive Care Department, UZ Brussels VUB University I0I, Laarbeeklaan, Jette 1090, Brussels, Belgium

Tel +3224749097

Fax +32 24775253

Email patrick.honore@uzbrussel.be (c) (1) (\$) $\odot 2015$ Honore et al. This work is published by Dove Medical Press Limited, and licensed under Creative Commons Attribution - Non Commercial (unported, v3.0)

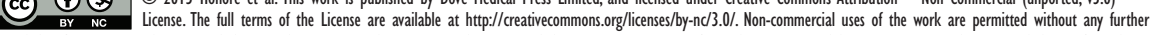
permissson Wove Medical Press Limited, provided the work is properly attributed. Permissions beyond the scope of the License are administered by Dove Medical Press Limited. Information on how to request permission may be found at http://www.dovepress.com/permissions.php 
Among them, p-cresyl sulfate (PCS) represents one of the most discriminating biomarkers of uremia. ${ }^{6-8}$ Serum PCS concentrations depend on intestinal uptake of p-cresol, PCS metabolism, and renal PCS excretion. ${ }^{9}$ Renal dysfunction leads to accumulation of PCS, mainly because of decreased active tubular secretion. ${ }^{9}$ In CKD patients, serum PCS concentrations are independently linked and directly proportional to cardiovascular disease progression and overall mortality. ${ }^{6-8}$

Accordingly, reducing PCS levels might offer protection against cardiovascular and/or ischemic events in these patients. PCS has a molecular weight of $188 \mathrm{Da}$, a large volume of distribution, and high protein-binding. Thus, convective removal will eliminate PCS more effectively than peritoneal or high-flux intermittent hemodialysis. ${ }^{10}$ Other purification techniques (eg, fractionated plasma separation and adsorption $)^{11}$ or specific dialysis membranes ${ }^{12}$ could be applied to amplify removal, but studies confirming their benefit are still awaited. Albeit attractive, the p-cresyl sulfate hypothesis, and by extension all other "uremic toxin theories", still lack convincing evidence. Intestinal microbiota in patients with CKD becomes progressively dominated by bacterial phyla that degrade substantially more proteins than carbohydrates. $^{13}$

This flora produces an array of metabolites that used to be called uremic toxins. Recent publications ${ }^{14,15}$ underline that CKD contributes to increased gut permeability. Subsequent bacterial translocation then induces chronic inflammation that is further enhanced during hemodialysis. Whether these toxin-mediated and inflammation-mediated gut processes are associated with an increased risk of AEN can be challenged since they remain restricted to the distal part of the small bowel and colon. Yet, in contrast with healthy individuals, patients with CKD may develop bacterial colonization of the esophagus. ${ }^{16}$

\section{Indoxyl sulfate}

Indoxyl sulfate is the metabolite of the common amino acid tryptophan. Indoxyl sulfate also contributes to progression of CKD by inducing an inflammatory reaction characterized by enhanced expression of profibrotic cytokines, such as transforming growth factor beta $1 .{ }^{17}$ Indoxyl sulfate also accentuates endothelial dysfunction by generating oxidative stress and inducing endothelial senescence. ${ }^{18}$

Both P-cresyl and indoxyl sulfate are poorly eliminated by commonly used dialysis techniques and thus accumulate during the progression of CKD. ${ }^{17}$ Although they are considered to be pathophysiologic players and not simple biomarkers, ${ }^{6-18}$ their individual potential to cause cardiovascular harm in
CKD is difficult to estimate. Differing toxicity might be linked to a proportional difference of free and total toxin fraction due to differences in affinity and/or competition in protein binding, ${ }^{17}$ but this remains to be proven.

\section{Endotoxin translocation Dormant gut endotoxin}

Cardiac and mesenteric perfusion undergo considerable changes in dialysis-dependent patients with CKD. Patients with CKD can suffer dramatic volume overload whilst intermittent hemodialysis in combination with ultrafiltration may repeatedly cause significant hemodynamic instability. Subsequent ischemic injury to vulnerable vascular beds promotes not only acute but also long-term cardiovascular damage and increases mortality. Despite preserved systemic blood pressure, long-term maintenance intermittent hemodialysis and ultrafiltration are associated with reduced splanchnic blood flow and mucosal ischemia.

Mesenteric ischemia tends to disrupt gut mucosal structure and function, which results in increased gut permeability. ${ }^{19} \mathrm{~A}$ huge amount of endotoxin lays dormant in the mesenteric bed. ${ }^{20}$ Ischemia-induced activation followed by translocation of this endotoxin through a leaky gut will generate or enhance local and systemic organ dysfunction. ${ }^{20}$ The degree of kidney injury is directly proportional to the level of circulating endotoxin. If insufficiently inactivated or cleared, perpetuating endotoxin aggression will foster hemodynamic instability, cardiac depression, kidney injury, and gut ischemia, and finally hasten mortality. ${ }^{21}$ Endotoxin-linked cardiovascular and ischemic events in CKD patients may be counteracted by using highly adsorbing dialysis membranes and specific sorbents that facilitate endotoxin clearance from the circulation. ${ }^{12}$ Therapies that modulate endotoxin production (eg, administration of probiotic Lactobacillus strains) are currently under investigation. ${ }^{22}$

\section{Other sources of endotoxin}

Endotoxin may become sequestrated during intermittent hemodialysis as an effect of monocyte activation, which is commonly observed during extracorporeal circulation, or by direct adsorption on the dialysis membrane. Some material used in dialysis membranes like polyacrylonitrile (PAN) or polymethylmethacrylate (PMMA) have a potent ability to adsorb a wide variety of circulating substances, including endotoxin, deriving its high biocompatibility grade from the capacity to buffer complement and other factors within the reactive cascade. ${ }^{12,23}$ Exceptionally, endotoxin may also emanate from water purification systems. 


\section{Altered mucosal defense mechanisms \\ Reduced nitric oxide production}

The mortality of upper gastrointestinal hemorrhage is higher in patients with CKD than in patients with normal renal function. Bleeding diathesis in CKD has been attributed primarily to the mucosal vascular malformations and peptic ulcerations that are frequently observed in this population. Since CKD in itself does not cause gastric acid hypersecretion, other mechanisms must be implicated in the development of mucosal ischemia. Nitric oxide (NO), a molecule synthesized from L-arginine, plays an important role in mucosal defense. NO exerts "vasoprotective" effects by enhancing endothelial cell survival and proliferation, inhibiting excessive proliferation of vascular smooth muscle cells, and suppressing adhesion of platelets and inflammatory cells to the vessel wall..$^{24,25} \mathrm{NO}$ has been identified as a mediator of bleeding tendency in uremic rodents. Specific NO inhibition in these animals normalizes bleeding time. ${ }^{24}$

Asymmetric dimethylarginine (ADMA) is an endogenous inhibitor of NO synthase. Topical application of ADMA causes marked arterial vasoconstriction and endothelial dysfunction. ${ }^{26} \mathrm{ADMA}$ is recognized as a risk factor for developing cerebral and coronary atherosclerotic vascular disease and may jeopardize gut mucosal defenses by reducing mesenteric flow. ${ }^{25,26}$ ADMA levels were found to be significantly higher in dialysis patients than in normal controls. ${ }^{25-27}$

Of note is that various mechanisms may counterbalance the deleterious effects of insufficient NO production at the gastric mucosal level. Hypertension and anemia, which are common in CKD patients, tend to increase gastric blood flow. Vasodilating substances, such as adrenomedullin and atrial natriuretic peptide, may actively increase submucosal blood flow. In some patients, volume expansion will enhance gastric blood flow and avoid the occurrence of mucosal lesions. ${ }^{25-28}$

\section{"Denervation hypersensitivity" to nitric oxide}

The autonomic and immune system act in concert to protect the gut from ischemic insults. In this context, CKD patients undergoing dialysis often develop "denervation hypersensitivity" to NO. Low basal NO production may upregulate overall $\mathrm{NO}$ sensitivity and produce an exaggerated physiologic response to stimuli, ${ }^{24}$ in particular to hypoxia. ${ }^{29}$ Under these circumstances, alterations of non-adrenergic and non-cholinergic neurotransmitters (eg, vasoactive intestinal polypeptide and substance $\mathrm{P}$, displaying respectively an inhibitory and an excitatory effect) may play a pivotal role. The most dreaded effect is increased blood flow in already well-perfused gut areas whilst flow in less-perfused zones will fall, thereby aggravating local ischemia. ${ }^{29}$

Of course, any ischemic effect of the above described pathways may be enhanced or accelerated by other factors whether or not related to CKD or its treatment (eg, intercurrent infection and repeated episodes of hypotension during intermittent hemodialysis).

\section{Conclusion}

Recent case presentations of AEN in CKD patients undergoing hemodialysis encouraged us to look beyond hypoperfusion/ ischemia as the most accepted pathophysiologic substrate underlying this observation. We focused on three interesting pathways (protein-bound uremic toxins [p-cresyl sulfate and indoxyl sulfate], endotoxin translocation, and defective mucosal barrier function). Evidence supporting involvement of all these pathways in promoting gut ischemia in CKD patients undergoing dialysis is gradually accumulating. However, many aspects regarding these pathways (ie, their individual contributions to the ischemic process, eventual interplay, and therapeutic modulation) remain to be elucidated.

\section{Disclosure}

The authors declare no conflicts of interest in this work.

\section{References}

1. Haviv YS, Reinus C, Zimmerman J. "Black esophagus": A rare complication of shock. Am J Gastroenterol. 1996;91:2432-2434.

2. Mangan TF, Colley AT, Wytock DH. Antibiotic-associated acute necrotizing esophagitis. Gastroenterology. 1990;99:900

3. Moretó M, Ojembarrena E, Zaballa M, Tánago JG, Ibánez S. Idiopathic acute esophageal necrosis: not necessarily a terminal event. Endoscopy. $1993 ; 25: 534-538$

4. Lacy BE, Toor A, Bensen SP, Rothstein RI, Maheshwari Y. Acute esophageal necrosis: report of two cases and a review of the literature. Gastrointest Endosc. 1999;49:527-532

5. Butt MD, Luck NH, Hassan SM, Abbas Z, Mubarak M. Acute eosophageal necrosis in a patient with end stage renal disease and hemodialysis J Transl Intern Med. 2014;3:25-

6. Meijers BK, Bammens B, De Moor B, Verbeke K, Vanrenterghem Y, Evenepoel P. Free p-cresol is associated with cardiovascular disease in hemodialysis patients. Kidney Int. 2008;73:1174-1180

7. Meijers BK, Claes K, Bammens, et al. P-cresol and cardiovascular risk in mild to moderate kidney disease. Clin J Am Soc Nephrol. 2010;5: $1182-1189$

8. Bammens B, Evenepoel P, Keuleers H, Verbeke K, Vanrenterghem Y. Free serum concentrations of the protein-bound retention solute p-cresol predict mortality in hemodialysis patients. Kidney Int. 2006;69 1081-1087.

9. Poesen R, Viaene L, Verbeke K, et al. Cardiovascular disease relates to intestinal uptake of $\mathrm{p}$-cresol in patients with chronic kidney disease. BMC Nephrol. 2014;15:87. 
10. Bammens B, Evenepoel P, Verbeke K, et al. Removal of the proteinbound solute $\mathrm{p}$-cresol by convective transport: a randomized crossover study. Am J Kidney Dis. 2004;44:278-285.

11. Meijers BK, Weber V, Bammens B, et al. Removal of the uremic retention solute p-cresol using fractionated plasma separation and adsorption. Artif Organs. 2008;32:214-219.

12. Honore PM, Jacobs R, Joannes-Boyau O, et al. Newly designed CRRT Membranes for sepsis and SIRS - a pragmatic approach for bedside intensivists summarizing the more recent advances: a systematic structured review. ASAIO J. 2013;59:99-106.

13. Sirich TL, Aronov PA, Plummer NS, Hostetter TH, Meyer TW. Numerous protein-bound solutes are cleared by the kidney with high efficiency. Kidney Int. 2013;84:585-590.

14. Lin CJ, Pan CF, Chuang CK, et al. P-cresyl sulfate is a valuable predictor of clinical outcomes in pre-ESRD patients. Biomed Res Int. 2014;2014:526932.

15. Wu IW, Hsu KH, Hsu HJ, et al. Serum free p-cresyl sulfate levels predict cardiovascular and all-cause mortality in elderly hemodialysis patients - a prospective cohort study. Nephrol Dial Transplant. 2012;27: 1169-1175.

16. Shi K, Wang F, Jiang H, et al. Gut bacterial translocation may aggravate microinflammation in hemodialysis patients. Dig Dis Sci. 2014;59: 2109-2117.

17. Wu IW, Hsu KH, Lee CC, et al. P-Cresyl sulphate and indoxyl sulphate predict progression of chronic kidney disease. Nephrol Dial Transplant. 2011;26:938-947.

18. Yu M, Kim YJ, Kang DH. Indoxyl sulfate-induced endothelial dysfunction in patients with chronic kidney disease via an induction of oxidative stress. Clin J Am Soc Nephrol. 2011;6:30-39.

19. Jakob SM, Ruokonen E, Vuolteenaho O, Lampainen E, Takala J. Splanchnic perfusion during hemodialysis: evidence for marginal tissue perfusion. Crit Care Med. 2001;29:1393-1398.
20. Rachoin JS, Schorr CA, Dellinger RP. Targeting endotoxin in the treatment of sepsis. Subcell Biochem. 2010;53:323-338.

21. McIntyre CW, Harrison LE, Eldehni MT, et al. Circulating endotoxemia: a novel factor in systemic inflammation and cardiovascular disease in chronic kidney disease. Clin J Am Soc Nephrol. 2011;6:133-141.

22. Bajaj JS, Heuman DM, Hylemon PB, et al. Randomised clinical trial: Lactobacillus GG modulates gut microbiome, metabolome and endotoxemia in patients with cirrhosis. Aliment Pharmacol Ther. 2014;39: 1113-1125.

23. McIntyre CW, Harrison LE, Eldehni MT, et al. Circulating endotoxemia: a novel factor in systemic inflammation and cardiovascular disease in chronic kidney disease. Clin J Am Soc Nephrol. 2011;6:133-141.

24. Remuzzi G, Perico N, Zoia C, Macconi D, Vigano G. Role of endothelium-derived nitric oxide in the bleeding tendency of uremia. J Clin Invest. 1990;86:1768-1771.

25. Ugurcu V, Vatansev V, Unlu A, et al. Levels of arginine and its products in dialysis patients. Eur Rev Med Pharmacol Sci. 2014;16: 2357-2364.

26. Faraci FM, Brian JE, Heistad DD. Response of cerebral blood vessels to an endogenous inhibitor of nitric oxide synthase. Am J Physiol. 1995;269:1522-1527.

27. Abdeni S, Meinitzer A, Holme I, et al. Asymmetrical dimethylarginine is associated with renal and cardiovascular outcomes and all cause mortality in renal transplant recipients. Kidney Int. 2010;77:44-50.

28. Tomikawa M, Ohta M, Vaziri ND, et al. Decreased endothelial nitric oxide synthase in gastric mucosa of rats with chronic renal failure. $\mathrm{Am}$ J Physiol. 1998;274:1102-1108.

29. Matteoli G, Boeckxstaens GE. The vagal innervation of the gut and immune homeostasis. Gut. 2013;62:1214-1222.

\section{Publish your work in this journal}

The International Journal of Nephrology and Renovascular Disease is an international, peer-reviewed open-access journal focusing on the pathophysiology of the kidney and vascular supply. Epidemiology, screening, diagnosis, and treatment interventions are covered as well as basic science, biochemical and immunological studies. The journal welcomes original research, clinical studies, reviews \& evaluations, expert opinion and commentary, case reports and extended reports. The manuscript management system is completely online and includes a very quick and fair peerreview system, which is all easy to use. Visit http://www.dovepress.com/ testimonials.php to read real quotes from published authors. 